\title{
The Higgs Boson in the Periodic System of Elementary Particles
}

\author{
Ding-Yu Chung ${ }^{1}$, Ray Hefferlin ${ }^{2}$ \\ ${ }^{1}$ Utica, Michigan, USA \\ ${ }^{2}$ Department of Physics, Southern Adventist University, Collegedale, USA \\ Email: dy_chung@yahoo.com, hefferln@southern.edu \\ Received February 12, 2013; revised March 15, 2013; accepted March 27, 2013
}

Copyright (C) 2013 Ding-Yu Chung, Ray Hefferlin. This is an open access article distributed under the Creative Commons Attribution License, which permits unrestricted use, distribution, and reproduction in any medium, provided the original work is properly cited.

\begin{abstract}
It is proposed that the observed Higgs Boson at the LHC is the Standard Model Higgs boson that adopts the existence of the hidden lepton condensate. The hidden lepton is in the forbidden lepton family, outside of the three lepton families of the Standard Model. Being forbidden, a single hidden lepton cannot exist alone; so it must exist in the lepton condensate as a composite of $\mu^{\prime}$ and $\mu^{, \pm}$hidden leptons and their corresponding antileptons. The calculated average mass of the hidden lepton condensate is $128.8 \mathrm{GeV}$ in good agreements with the observed 125 or $126 \mathrm{GeV}$. The masses of the hidden lepton condensate and all elementary particles including leptons, quarks, and gauge bosons are derived from the periodic system of elementary particles. The calculated constituent masses are in good agreement with the observed values by using only four known constants: the number of the extra spatial dimensions in the eleven-dimensional membrane, the mass of electron, the mass of $\mathrm{Z}$ boson, and the fine structure constant.
\end{abstract}

Keywords: Higgs Boson; Periodic System; Elementary Particles

\section{Introduction}

As described in "In Molecular Taxonomy: String, quark, hadron, nuclear, atomic and chemical molecule periodic or invariant systems" [1], chemists and physicists have accumulated vast libraries of data about the properties and atoms and molecules via the simplest to the most complex experiments; from Bohr to Schrödinger theory; and using back-of-the-envelope to supercomputer computation. Much of it is distilled into the periodic chart of the elements and models about the various molecular bonds. Though starting more recently, scientists have garnered as many or more numbers describing characteristics of nuclei, the essence of which is seen in the chart of the isotopes. Still more recently, the simplest fundamental particle properties have been epitomized in the geometric patterns showing hadrons and their quark constituents. And now even string theory has produced a periodic system, such as the periodic system of elementary particles $[2,3]$.

At the LHC (Large Hadron Collider), the ATLAS and CMS experiments of CERN experiments observe a new particle in the mass region around $125-126 \mathrm{GeV}$ with a high degree of certainty, and the decay modes and the spin of the new particle point to the Standard Model
Higgs boson [4,5].

In this paper, the periodic system of elementary particles based on string theory is used to describe the newly discovered Higgs boson at the LHC, and the calculated mass from this periodic system of elementary particles is in good agreement with the observed value.

\section{The Periodic System of Elementary Particles}

The periodic system of elementary particles is based on the "überparticles" of our universe. An überparticle consists of two four dimensional particles each with its own set of seven "dimensional orbitals". The seven dimensional orbitals represent originally [6] the seven extra spatial dimensions in the eleven dimensional membrane of string theory.

In an überparticle, one four dimensional particle has the seven "principal dimensional orbitals", while the other four dimensional particle has the seven "auxiliary dimensional orbitals" that are superimposed on the seven principal dimensional orbitals. In an überparticle, the auxiliary orbitals are dependent on the principal orbitals, so only one set of dimensional orbitals appears. The principal dimensional orbitals are mainly for leptons and 
gauge bosons, and the auxiliary dimensional orbitals are mainly for individual quarks. Because of the dependence of the auxiliary dimensional orbitals, individual quarks are hidden. The configuration of dimensional orbitals and the periodic system of elementary particles are shown in Figure 1 and Table 1.

Figure 1 shows leptons and quarks. The symbols of the top row represent leptons and their components. $v_{e}, e$, $v_{\mu}$, and $v_{\tau}$ are on the principal dimensional orbitals, 5,6 , 7 , and 8 , respectively. Other leptons $\left(\mu, \tau\right.$, and $\left.\mu^{\prime}\right)$ are the composites of both the principal components ( $e$ and $v$ ) and auxiliary components $\left(\mu_{7}, \tau_{7}\right.$ and $\left.\mu_{8}\right)$ as shown in Table 2. The symbols below the numbers are the principal and auxiliary components of quarks. All quarks are the composites of both principal components $\left(u_{5}, d_{6}, \mu\right.$, and $\left.\mu^{\prime}\right)$ and auxiliary components $\left(u_{7}, d_{7}, s_{7}, c_{7}, b_{7}, t_{7}, b_{8}\right.$, and $t_{8}$ ) as shown in Table 2 . The seven auxiliary dimensional orbitals can accommodate only three families of lepton-quark as in the Standard Model. No leptons and quarks appear on and higher than $d=9$, which is outside of the Standard Model and the seven auxiliary dimensional orbitals. $f_{9}$ and $f_{10}$ are the principal fermions on $d=$ 9 and 10, respectively. The gauge bosons are not shown, and can be located on the top row at principal dimensional orbitals as shown in Table 1.

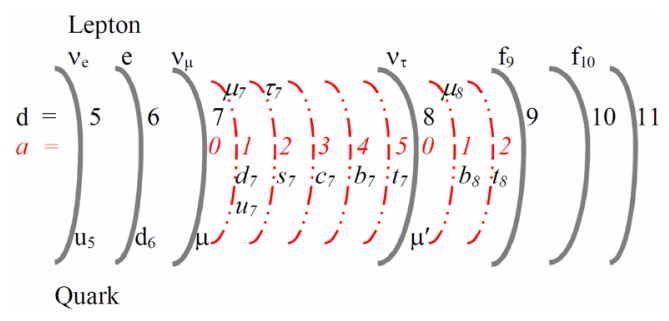

Figure 1. The überparticle represented by leptons and quarks in the seven principal dimensional orbitals (solid lines) denoted by the principal dimensional orbital number $d$ and the seven auxiliary dimensional orbitals (dash-dotted lines) denoted by the auxiliary dimensional number $a$.

Table 1. The überparticle represented by the periodic system of elementary particles $d$ = principal dimensional orbital number, $a$ = auxiliary dimensional orbital number.

\begin{tabular}{|c|c|c|c|c|c|c|c|c|c|c|}
\hline$d$ & $a=0$ & 1 & 2 & $a=0$ & 1 & 2 & 3 & 4 & 5 & \\
\hline & Lepton & & & Quark & & & & & & Boson \\
\hline 5 & $l_{5}=v_{e}$ & & & $q_{5}=u_{5}$ & & & & & & $B_{5}=A$ \\
\hline 6 & $l_{6}=e$ & & & $q_{6}=d_{6}$ & & & & & & $B_{6}=\pi_{1 / 2}$ \\
\hline 7 & $l_{7}=v_{\mu}$ & $\mu_{7}$ & $\tau_{7}$ & $q_{7}=\mu$ & $u_{7} / d_{7}$ & $s_{7}$ & $c_{7}$ & $b_{7}$ & $t_{7}$ & $B_{7}=Z_{L}^{0}$ \\
\hline 8 & $l_{8}=v_{\tau}$ & $\mu_{8}$ & & $q_{8}=\mu^{\prime}$ (hidden) & $b_{8}$ (hidden) & $t_{8}$ & & & & $B_{8}=X_{R}$ \\
\hline 9 & $f_{9}$ & & & & & & & & & $B_{9}=X_{L}$ \\
\hline 10 & $f_{10}$ & & & & & & & & & $B_{10}=Z_{R}^{0}$ \\
\hline 11 & & & & & & & & & & $B_{11}$ \\
\hline
\end{tabular}

Table 2. The compositions and the constituent masses of leptons and quarks $d=$ principal dimensional orbital number and $a$ = auxiliary dimensional orbital number.

\begin{tabular}{|c|c|c|c|}
\hline & $d_{a}$ & Composition & Calculated Mass \\
\hline Leptons & $\underline{d_{a}}$ for leptons & & \\
\hline$v_{e}$ & $5_{0}$ & $v_{e}$ & $\approx 0$ \\
\hline$e$ & $6_{0}$ & $e$ & $0.51 \mathrm{MeV}$ (given) \\
\hline$v_{\mu}$ & $7_{0}$ & $v_{\mu}$ & $\approx 0$ \\
\hline$v_{\tau}$ & $8_{0}$ & $v_{\tau}$ & $\approx 0$ \\
\hline$\mu$ & $6_{0}+7_{0}+7_{1}$ & $e+v_{\mu}+\mu_{7}$ & $105.6 \mathrm{MeV}$ \\
\hline$\tau$ & $6_{0}+7_{0}+7_{2}$ & $e+v_{\mu}+\tau_{7}$ & $1786 \mathrm{MeV}$ \\
\hline$\mu^{\prime}$ & $6_{0}+7_{0}+7_{2}+8_{0}+8_{1}$ & $e+v_{\mu}+\mu_{7}+v_{\tau}+\mu_{8\left(3 / 2 Z^{\circ}\right)}$ & $136.9 \mathrm{GeV}$ \\
\hline$\mu^{ \pm \pm}$ & $6_{0}+7_{0}+7_{2}+8_{0}+8_{1}^{ \pm}$ & $e+v_{\mu}+\mu_{7}+v_{\tau}+\mu_{8}^{ \pm}\left(3 / 2 W^{ \pm}\right)$ & $120.7 \mathrm{GeV}$ \\
\hline Quarks & $\underline{d_{a}}$ for quarks & & \\
\hline$u$ & $5_{0}+7_{0}+7_{1}$ & $q_{5}+q_{7}+u_{7}$ & $330.8 \mathrm{MeV}$ \\
\hline$d$ & $6_{0}+7_{0}+7_{1}$ & $q_{6}+q_{7}+d_{7}$ & $332.3 \mathrm{MeV}$ \\
\hline$s$ & $6_{0}+7_{0}+7_{2}$ & $q_{6}+q_{7}+s_{7}$ & $558 \mathrm{MeV}$ \\
\hline$c$ & $5_{0}+7_{0}+7_{3}$ & $q_{5}+q_{7}+c_{7}$ & $1701 \mathrm{MeV}$ \\
\hline$b$ & $6_{0}+7_{0}+7_{4}$ & $q_{6}+q_{7}+b_{7}$ & $5318 \mathrm{MeV}$ \\
\hline$t$ & $5_{0}+7_{0}+7_{5}+8_{0}+8_{2}$ & $q_{5}+q_{7}+t_{7}+q_{8}+\mathrm{t}_{8}$ & $176.5 \mathrm{GeV}$ \\
\hline
\end{tabular}


Table 1 shows leptons, quarks, and gauge bosons. For leptons, the leptons at $a=0$ are the principal leptons ( $v_{e}$, $e, v_{\mu}$, and $\left.v_{\tau}\right)$. Other leptons $\left(\mu, \tau\right.$, and $\left.\mu^{\prime}\right)$ are the composites of both the principal components ( $e$ and $v$ at $a=0$ ) and auxiliary components $\left(\mu_{7}, \tau_{7}\right.$ and $\mu_{8}$ at $\left.a>0\right)$ as shown in Table 2. All quarks are the composites of both principal components $\left(u_{5}, d_{6}, \mu\right.$, and $\mu^{\prime}$ at $\left.a=0\right)$ and auxiliary components $\left(u_{7}, d_{7}, s_{7}, c_{7}, b_{7}, t_{7}, b_{8}\right.$, and $t_{8}$ at $a>$ 0 ) as shown in Table 2. Outside of the Standard Model, $\mu^{\prime}$ is hidden, and is balanced by the hidden $b_{8}$. No leptons and quarks appear on and higher than $d=9$, which is outside of the Standard Model and the seven auxiliary dimensional orbitals. The bosons, $B_{5}, B_{6}, B_{7}, B_{8}, B_{9}, B_{10}$, and $B_{11}$ are shown in Table 3 .

The principal dimensional orbitals are for gauge bosons of the force fields, as shown in Table 1. It will be shown shortly that $\mathrm{F}_{\mathrm{d}}$ has lower energy than $B_{d}$. and similarly the seven principal dimensional orbitals are arranged as $F_{5} B_{5} F_{6} B_{6} F_{7} B_{7} F_{8} B_{8} F_{9} B_{9} F_{10} B_{10} F_{11} B_{11}$, where $B$ and $F$ are the boson and fermion in each orbital. In previous communications [3] we have shown that the masses of fundamental particles are related to each other with three simple formulae, and that the use of accepted mass data allows calculation of masses of many other particles. The formulae are

$$
\begin{gathered}
M_{d, F}=M_{d, B} \alpha_{d, B}, \\
M_{d-1, B}=M_{d, F} \alpha_{d, F}, \\
M_{d-1, B}=M_{d, B} \alpha_{d}^{2},
\end{gathered}
$$

where $d$ is the dimensional orbital number from 6 to 11 . $E_{5, B}$ and $E_{11, B}$ are the energies for the $5 d$ dimensional orbital and the $11 d$ dimensional orbital, respectively. Each dimension has its own $\alpha_{d}$, and all $\alpha_{d}^{\prime}$ s except $\alpha_{7}\left(\alpha_{w}\right)$ of the seventh dimension (weak interaction) are equal to $\alpha$, the fine structure constant of electromagnetism. The lowest energy boson is the Coulombic field for electromagnetism and the second lowest boson energy is $\pi_{1 / 2}$ (a spin 1 boson as a half of the spin 0 pion) for the strong interaction.

$$
\begin{aligned}
& M_{5, B}=\alpha M_{6, F}=\alpha M_{e} . \\
& M_{6, B}=M_{5, B} / \alpha^{2}=M_{e} / \alpha=M_{\pi / 2}
\end{aligned}
$$

The bosons generated in this manner are the dimensional orbital bosons or $B_{D}$ as shown in Table 3 .

In Table 3, $\alpha=\alpha_{e}$ (the fine structure constant for electromagnetic field), $M_{Z}=M_{w} / \cos q_{w}$, and $\alpha_{w}=\alpha / \sin ^{2} q_{w}$ [7]. $\alpha_{w}$ is not same as $\alpha_{e}$ because there is a mixing between $\mathrm{B}_{5}$ and $\mathrm{B}_{7}$ as the symmetry mixing between $\mathrm{U}(1)$ and $\mathrm{SU}(2)$ in the standard theory of the electroweak interaction, and $\sin \theta_{w}$ is not equal to 1 . The calculated value for $\alpha_{w}$ is 0.02973 , and $\sin ^{2} \theta_{w}$ is 0.2454 in good agreement with 0.2312 for the observed value of $\sin ^{2} \theta_{w}$ [8]. The calculated energy for $B_{11}$ is $1.1 \times 10^{19} \mathrm{GeV}$ in good agreement with the Planck mass, $1.2 \times 10^{19} \mathrm{GeV}$. The strong interaction, represented by $\pi_{1 / 2}$ (half of a pion), is for the interactions among quarks and for the hiding of individual quarks in auxiliary orbitals. The weak interaction, represented by $Z_{L}^{0}$, is for the interaction involving changing flavors among quarks and leptons. The auxiliary dimensional orbitals are derived from principal dimensional orbitals. They are for high-mass leptons and individual quarks.

All leptons and quarks with $d$ 's, $a$ 's and the calculated masses are listed in Table 2 as discussed in Ref. [3]

Table 2 shows the compositions of leptons and quarks. Each fermion can be defined by principal dimensional orbital numbers ( $d$ 's) and auxiliary dimensional orbital numbers ( $a$ 's). For examples, $e$ is $6_{0}$ that means it has $\mathrm{d}$ (principal dimensional orbital number) $=6$ and a (auxiliary dimensional orbital number) $=0$, so e is a principal dimensional fermion. All neutrinos are nearly massless because of chiral symmetry (permanent chiral symmetry). As shown in Table 2, $\mu^{\prime}$ and $\mu^{\prime \pm}$ are the hidden (forbidden) leptons outside of the three lepton families in the Standard Model. All elementary particles (gauge bosons, leptons, and quarks) are in the periodic system of elementary particles with the calculated constituent masses in good agreement with the observed values $[9,10]$ by using only four known constants: the number of the extra

Table 3. The Masses of the dimensional orbital gauge bosons: $\alpha=\alpha_{e}, d=$ dimensional orbital number.

\begin{tabular}{ccccc}
\hline $\mathrm{B}_{\mathrm{d}}$ & $M_{d}$ & $\mathrm{GeV}$ (calculated) & Gauge boson & Interaction \\
\hline $\mathrm{B}_{5}$ & $M_{e} \alpha$ & $3.7 \times 10^{-6}$ & $\mathrm{~A}=$ photon & Electromagnetic \\
$\mathrm{B}_{6}$ & $M_{e} / \alpha$ & $7 \times 10^{-2}$ & $\pi_{1 / 2}$ & Strong \\
$\mathrm{B}_{7}$ & $M_{6} / \alpha_{w}^{2} \cos \theta_{w}$ & 91.177 (given) & $Z_{L}^{0}$ & weak (left) \\
$\mathrm{B}_{8}$ & $M_{7} / \alpha^{2}$ & $1.7 \times 10^{6}$ & $X_{R}$ & $\mathrm{CP}$ (right) nonconservation \\
$\mathrm{B}_{9}$ & $M_{8} / \alpha^{2}$ & $3.2 \times 10^{10}$ & $X_{L}$ & $\mathrm{CP}$ (left) nonconservation \\
$\mathrm{B}_{10}$ & $M_{9} / \alpha^{2}$ & $6.0 \times 10^{14}$ & $Z_{R}^{0}$ & weak (right) \\
$\mathrm{B}_{11}$ & $M_{10} / \alpha^{2}$ & $1.1 \times 10^{19}$ & $\mathrm{G}$ & Gravity \\
\hline
\end{tabular}


spatial dimensions in the eleven-dimensional membrane, the mass of electron, the mass of $Z$ boson, and the fine structure constant. For an example, the calculated mass of top quark is $176.5 \mathrm{GeV}$ in good agreement with the observed $173.5 \mathrm{GeV}$ [11].

\section{The Observed Higgs Boson}

We propose that the observed Higgs boson is the Standard Model Higgs boson that adopts the existence of the hidden lepton condensate. We describes the hidden lepton condensate first, and the adoption of the hidden lepton condensate later. The hidden lepton condensate is similar to the top quark condensate. The top quark condensate is a composite field composed of the top quark and its antiquark. The top quark condenses with its measured mass (173 GeV) comparable to the mass of the $\mathrm{W}$ and Z Bosons, so Vladimir Miransky, Masaharu Tanabashi, and Koichi Yamawaki [12] proposed that the top quark condensate is responsible for the mass of the $\mathrm{W}$ and $\mathrm{Z}$ bosons. The top quark condensate is analogous to Cooper pairs in a BCS superconductor and nucleons in the Nambu-Jona-Lasinio model [13]. Anna Hasenfratz and Peter Hasenfratz et al. [14] claimed that the top quark condensate is approximately equivalent to a Higgs scalar field. S. F. King proposed a tau lepton condensate to feed the tau mass to the muon and the electron $[15,16]$.

By analogy to the top quark condensate, the hidden lepton condensate is a field composed of the hidden leptons and antileptons $\mu^{\prime}, \bar{\mu}^{\prime}, \mu^{\prime \pm}$, and $\bar{\mu}^{ \pm}$as shown in Table 2. Just as the observed top quark is a bare quark with the observed mass of about $173 \mathrm{GeV}$ instead of about $346 \mathrm{GeV}$ for top quark-antitop quark, the observed hidden lepton is a bare average hidden lepton instead of $\mu^{\prime}, \bar{\mu}^{\prime}$ and $\mu^{\prime \pm}, \bar{\mu}^{\prime \pm}$. However, unlike the top quark condensate, the hidden lepton condensate is outside of the standard three lepton-quark families in the Standard Model. Being forbidden, a single hidden lepton cannot exist alone, so the hidden leptons must exist in the lepton condensate as the composite of the leptons-antileptons $\mu^{\prime}, \bar{\mu}^{\prime}, \mu^{\prime \pm}$, and $\bar{\mu}^{\prime \pm}$.

To match $l_{8}\left(v_{\tau}\right)$ at $d=8$, quarks include $q_{8}$ at $d=8$ as a part of the $t$ quark. In the same way that $q_{7}=3 \mu, q_{8}$ involves $\mu^{\prime} . \mu^{\prime}$ is the sum of $e, \mu$, and $\mu_{8}$ (auxiliary dimensional leptons). Using Equation (14) of Ref. [3], the mass of $\mu_{8}$ equals to $3 / 2$ of the mass of $B_{7}$, which is $Z^{0}$, and the mass of $\mu_{8}^{ \pm}$equals to $3 / 2$ of the mass of $B_{7}^{ \pm}$, which is $W^{ \pm}$. From these we can calculate the masses of $\mu^{\prime}$ and $\mu^{\prime \pm}$.

$$
\begin{aligned}
\mu_{8} & =\frac{3}{2} Z^{0} \\
\mu^{\prime} & =6_{0}+7_{0}+7_{2}+8_{0}+8_{1} \\
& =e+v_{\mu}+\mu_{7}+v_{\tau}+\mu_{8}
\end{aligned}
$$

$$
\begin{aligned}
\mu_{8}^{ \pm} & =\frac{3}{2} W^{ \pm} \\
\mu^{\prime \prime \pm} & =6_{0}+7_{0}+7_{2}+8_{0}+8_{1}^{ \pm} \\
& =e+v_{\mu}+\mu_{7}+v_{\tau}+\mu_{8}^{ \pm}
\end{aligned}
$$

The calculated masses of the hidden lepton are 120.7 $\mathrm{GeV}$ (for the mass of $\mu^{\prime \pm}$ ) and $136.9 \mathrm{GeV}$ (for the mass of $\mu^{\prime}$ ) with the average as $128.8 \mathrm{GeV}$ for the hidden lepton condensate, in good agreements with the results from $\mathrm{LHC}(125 \mathrm{GeV}$ or $126 \mathrm{GeV})$.

It is proposed that the Higgs scalar boson itself is a virtual zero-energy scalar boson without permanent energy to avoid the cosmological constant problem from the huge gravitational effect by the non-zero-energy Higgs boson [17]. Without permanent energy, the Higgs boson emerges and disappears by borrowing energy from and returning energy to the particles in the electroweak interaction, respectively. The returning of energy from the Higgs scalar boson to the particles is through the absorption of the Higgs scalar boson by the particles. When a massless particle in the electroweak interaction absorbs the Higgs scalar boson, the Higgs scalar boson becomes the longitudinal component of the massless particle, resulting in the massive particle and the disappearance of the Higgs scalar boson.

The observed Higgs boson at the LHC is a remnant of the Higgs boson. At the beginning of the universe, all particles in the electroweak interaction were massless. The Higgs boson appeared by borrowing energy symmetrically from all particles in the electroweak interaction. The Higgs boson coupled with all massless particles, including leptons, quarks, and gauge bosons, in the electroweak interaction. All massless particles except photon as the gauge boson for electromagnetism absorbed the Higgs boson as their longitudinal components to become massive particles. The asymmetrical returning of energy from the Higgs boson by the absorption of the Higgs boson is called the symmetrical breaking of the electroweak interaction in the Standard Model. In the cases of such massive particles, including leptons, quarks, and weak gauge bosons, the Higgs boson disappeared. In the case of massless photon as the gauge boson of electromagnetism, the un-absorbed Higgs boson became the remnant of the Higgs boson.

Being specific to the electroweak interaction, the remnant of the Higgs boson could not return the borrowed energy to any other massless particles, so it adopted the existence of another scalar boson, and returned the pure borrowed energy without the longitudinal component to photon. Essentially, the remnant of the Higgs boson became an avatar as a life born in the form of another life. The remnant of the Higgs boson could not adopt the existence of any scalar boson that already represented an independent elementary particle. The only available sca- 
lar boson came from the scalar hidden lepton condensate. Consequently, the remnant of the Higgs boson as the Standard Model Higgs boson adopted the existence of the hidden lepton condensate, and became the Avatar Higgs boson.

The two possibilities for the Standard Model Higgs boson that adopts the hidden lepton condensate are the pure Standard Model Higgs boson and the dual particle consisting of the Standard Model Higgs boson and the non-Standard Model hidden lepton condensate. The decay of the pure Standard Model Higgs boson follows the decay modes of the Standard Model.

Being outside of the Standard Model of the electroweak interaction, the hidden lepton condensate cannot decay into the particles in the electroweak interaction, and decay into diphoton via the internal annihilation ofparticle-antiparticle. As a result, the decay of the dual particle generates excess diphoton deviated from the Standard Model. The experimental evidence for the excess diphoton is inconclusive [18-20].

\section{Summary}

It is proposed that all elementary particles including leptons, quarks, and gauge bosons can be placed in the periodic system of elementary particles. The periodicity is derived from the two sets of seven mass orbitals where seven comes from the seven extra dimensions in the eleven-dimensional membrane in the string theory. The calculated constituent masses are in good agreement with the observed values by using only four known constants: the number of the extra spatial dimensions in the eleven-dimensional membrane, the mass of electron, the mass of $\mathrm{Z}$ boson, and the fine structure constant.

It is proposed that the Higgs boson itself is a virtual zero-energy gauge boson by borrowing energy from and returning energy to the particles in the electroweak interaction. When a massless particle in the electroweak interaction absorbs the Higgs boson, the Higgs boson becomes the longitudinal component of the massless particle, resulting in the massive particle. At the beginning of the universe, all particles in the electroweak interaction were massless. The symmetrical borrowing of energy from the particles and the asymmetrical returning of energy by the absorption of the Higgs boson resulted in the asymmetrical breaking of electroweak interaction and the remnant of the Higgs boson from the absence of the absorption of the Higgs boson by photon. The remnant of the Higgs boson adopted the existence of the scalar hidden lepton condensate and returned the borrowed energy to photon.

The observed Higgs Boson at the LHC is the Standard Model Higgs boson that adopts the existence of the hidden lepton condensate. The hidden lepton is in the for- bidden lepton family, outside of the three lepton families of the Standard Model. Being forbidden, a single hidden lepton cannot exist alone; so it must exist in the lepton condensate as a composite of $\mu^{\prime}$ and $\mu^{\prime \pm}$ hidden leptons and their corresponding antileptons. The calculated average mass of the hidden lepton condensate is $128.8 \mathrm{GeV}$ in good agreements with the observed 125 or $126 \mathrm{GeV}$.

\section{REFERENCES}

[1] R. Hefferlin, "Molecular Taxonomy: String, Quark, Hadron, Nuclear, Atomic and Chemical Molecule Periodic or Invariant Systems," LAP LAMBERT Academic Publishing, Saarbrücken, 2011.

[2] R. Hefferlin, "Molecular Taxonomy: String, Quark, Hadron, Nuclear, Atomic and Chemical Molecule Periodic or Invariant Systems," LAP LAMBERT Academic Publishing, Section 7, 2011. pp. 35-38

[3] D. Chung, "The Periodic System of Elementary Particles and the Composition of Hadrons," Speculations in Science and Technology, Vol. 20, 1997, pp. 259-268 http://arxiv.org/ftp/hep-th/papers/0111/0111147.pdf

[4] The ATLAS Collaboration, "Observation of a New Particle in the Search for the Standard Model Higgs Boson with the ATLAS Detector at the LHC," 2012. arXiv:1207.7235v1[hep-ex]

[5] The CMS Collaboration, "Observation of a New Boson at a Mass of $125 \mathrm{GeV}$ with the CMS Experiment at the LHC," 2012. arXiv:1207.7235v1[hep-ex]

[6] D. Chung and V. Krasnoholovets, "The Cosmic Organism Theory,” Scientific Inquiry, Vol. 8, 2007, pp. 165-182. http://www.iigss.net/Scientific-Inquiry/Dec07/3-krasnoho lovets.pdf

[7] A. Salam, "Weak and Electromagnetic Interactions," In: W. Svartholm, Ed., Elementary Particle Theory, Almquist and Wiksell, Stockholm, 1968, pp. 367-387.

[8] J. Beringe, et al., (Particle Data Group), "Electroweak Model and Constraints on New Physics," Physical Review D, Vol. 86, 2012, Article ID: 010001.

http://pdg.lbl.gov/2012/reviews/rpp2012-rev-standard-mo del.pdf

[9] D. Griffiths, "Introduction to Elementary Particles," WILEY-VCH, Hoboken, 2008, p. 135

[10] M. H. MacGregor, "Electron Generation of Leptons and Hadrons with Conjugate $\alpha$-Quantized Lifetimes and Masses," International Journal of Modern Physics A, Vol. 20, No. 4, 2005, pp. 719-798.

[11] J. Beringer, et al., (Particle Data Group), "Electroweak Model and Constraints on New Physics," Physical Review D, Vol. 86, 2012, Article ID: 010001. http://pdg.lbl.gov/2012/reviews/rpp2012-rev-top-quark.pd f

[12] V. A. Miransky, M. Tanabashi and K. Yamawaki, "Is the $t$ Quark Responsible for the Mass of $W$ and $Z$ Bosons," Modern Physics Letter A, Vol. 4, No. 11, 1989, pp. 10431063. doi:10.1142/S0217732389001210 
[13] Y. Nambu and G. Jona-Lasinio, "Dynamical Model of Elementary Particles Based on an Analogy with Superconductivity, II," Physical Review, Vol. 124, No. 1, 1961, pp. 246-254. doi:10.1103/PhysRev.124.246

[14] A. Hasenfratz, P. Hasenfratz, K. Jansen, J. Kuti and Y. Shen, "The Equivalence of the Top Quark Condensate and the Elementary Higgs Field," Nuclear Physics B, Vol. 365 , No. 1, 1991, pp. 79-97. doi:10.1016/0550-3213(91)90607-Y

[15] S. F. King, "Four-Family Lepton Mixing," Physical Review D, Vol. 46, 1992, pp. 4804-4807.

[16] S. F. King, "Low Scale Technicolour at LEP," Physics Letter B, Vol. 314, No. 3-4, 1993, pp. 364-370. doi:10.1016/0370-2693(93)91250-Q
[17] S. Weinberg, "The Cosmological Constant Problem," Review Modern Physics, Vol. 61, No. 1, 1989, pp. 1-23. doi:10.1103/RevModPhys.61.1

[18] P. P. Giardino, K. Kannike, M. Raidal and A. Strumia, "Is the Resonance at $125 \mathrm{GeV}$ the Higgs Boson?" 2012. arXiv:1207.1347v1 [hep-ph]

[19] K. R. Bland, (for the CDF, $\mathrm{D}_{0}$ Collaborations), "Search for the Higgs boson in H- $>\gamma \gamma$ Decays in $\$ p \backslash b a r\{p\} \$$ Collisions at $1.96 \mathrm{TeV}, " 2012$. arXiv:1110.1747v1[hep-ex]

[20] M. Strassler, "CMS Sees No Excess in Higgs Decays to Photons," 2013.

http://profmattstrassler.com/2013/03/14/cms-sees-no-exce ss-in-higgs-decays-to-photons/\#more-5655 